\title{
Identificación intraoperatoria del área motora suplementaria en cirugía neuro- oncológica
}

\author{
A. Gabarrós; J. Martino; M. Juncadella*; G. Plans; R. Pujol**; J. Deus**; O. Godino; A. Torres; A. Aparicio; G. \\ Conesa*** y J.J. Acebes \\ Servicios de Neurocirugía y Neuropsicología*. Hospital Universitari de Bellvitge (Barcelona). Servicios de Neurorradiología** y \\ Neurocirugía***. Hospital del Mar (Barcelona)
}

\section{Resumen}

Objetivos. El objetivo principal del estudio es conseguir la identificación intraoperatoria de la función del área motora suplementaria (AMS) implicada en tareas motoras complejas. El objetivo secundario es valorar el pronóstico funcional tras la preservación quirúrgica de este área.

Método. Se han seleccionado 15 pacientes con tumores cerebrales localizados en área premotora. Todos los pacientes fueron intervenidos despiertos. El córtex motor primario fue identificado mediante estimulación cerebral directa. Para identificar el AMS, el paciente realizó una tarea motora de oposición de dedos con la mano contralateral a la lesión que se bloqueaba mediante la estimulación eléctrica del córtex cerebral premotor.

Resultados. El AMS pudo ser identificada en todos los pacientes mediante este método.

La resección fue macroscópicamente completa en $\mathbf{1 3}$ pacientes $(86.6 \%)$ y subtotal en $2(13.3 \%)$. La función encontrada en el AMS se ha podido preservar en 14 pacientes $(93,3 \%)$. Sólo en una paciente se resecó parcialmente el AMS funcional junto a la lesión por infiltración de la misma (6.6\%). En el postoperatorio inmediato 8 pacientes $(53.3 \%)$ presentaron empeoramiento de los déficits, cinco pacientes $(33.3 \%)$ no mostraron cambios respecto a su exploración preoperatoria y 2 pacientes mejoraron. A los 6 meses de la intervención 5 pacientes $(33.3 \%)$ se encuentran asintomáticos y 10 pacientes presentan déficits permanentes. En este último grupo, 5 pacientes $(33.3 \%)$ muestran un déficit muy leve que no interfiere en su vida normal. En los otros 5 pacientes $(\mathbf{3 3 . 3} \%)$ los déficits permanentes interfieren con la vida del paciente: dos pacientes presentan hemiparesia severa 3/5 (igual estado preoperatorio que no mejora), uno afasia de expresión y otros dos $(\mathbf{1 3 . 3 \%})$ presentan un síndrome del AMS izquierda permanente. En los 2 pacientes con hemiparesia severa postoperatoria se

Recibido: 10-05-10. Aceptado: 12-09-10 objetivó intraoperatoriamente una infiltración tumoral del córtex motor primario y de la vía motora; el déficit motor severo preoperatorio (KPS $<70)$ se asoció con mal pronóstico funcional postoperatorio.

Conclusiones. La estimulación cortical intraoperatoria resulta útil para la identificación del AMS. La preservación del AMS, una vez identificada, disminuye la aparición de síntomas postquirúrgicos y la persistencia del síndrome del AMS. Cuando el AMS se encuentra infiltrada por tumor, su resección quirúrgica puede causar déficits permanentes, especialmente en el hemisferio dominante. El déficit motor severo preoperatorio se asocia con mal pronóstico funcional.

PALABRAS CLAVE: Área motora suplementaria. Mapping. Tarea intraoperatoria. Paciente despierto. Tumor cerebral

Intraoperative identification of the supplementary motor area in neurooncological surgery

Summary

Objectives. The main objective of the present work was to identify, by means of intraoperative electrical stimulation, the supplementary motor area (SMA) region which is implicated in complex motor function. The functional prognostic relevance of the surgical preservation of this area was also analyzed.

Method. Fifteen patients with tumors infiltrating the premotor cortex were selected. All patients were operated under awake conditions. Primary motor cortex was identified with intraoperative electrical stimulation (IES). To identify the SMA, patients were asked to do a finger opposition motor task with their hand contralateral to the lesion, that was blocked by electrically stimulating the premotor cerebral cortex.

Results. SMA was identified in all patients with

Abreviaturas. AMS: área motora suplementaria. IES: electrical stimulation. RMf: resonancia magnética funcional. SMA: supplementary motor area. 
IES. Complete surgical resection was achieved in 13 patients $(86.6 \%)$ and subtotal in 2 patients $(13.3 \%)$. SMA function was preserved in 14 patients $(93.3 \%)$. In only one patient the SMA was partially resected because of tumor infiltration $(6.6 \%)$. In the immediate postoperative period, 8 patients $(53.3 \%)$ did not show changes in comparison to their preoperative clinical status, and 2 patients improved. At 6 months follow up, 5 patients $(33.3 \%)$ were asymptomatic and 10 patients showed permanent deficits. In this last group, five patients $\mathbf{( 3 3 . 3 \% )}$ showed mild deficits that did not interfere with a normal life. In the other 5 patients $(33.3 \%)$, permanent deficits interfered with daily life activities: two patients presented severe hemiparesis $3 / 5$ (same similar to their preoperative status with no improvement), one patient had motor aphasia, and two other patients $(\mathbf{1 3 . 3 \%})$ showed permanent left SMA syndrome.

In two patients with severe postoperative hemiparesis, tumor infiltration of primary motor cortex and piramidal pathway was observed; severe preoperative motor deficit (KPS $<70$ ) was associated with poor functional outcome.

Conclusions. Intraoperative electrical cortical stimulation is useful to identify the SMA. Once identified, SMA preservation decreases the risk of postoperative symptoms and permanent SMA syndrome. When SMA is infiltrated by the tumor, radical resection may cause permanent neurological deficits, specially in the dominant hemisphere. Severe preoperative motor deficit was associated with poor outcome.

KEY WORDS: Supplementary motor area. Mapping. Intraoperative task. Awake patient Brain tumor.

\section{Introducción y objetivo}

El área motora suplementaria (AMS) se localiza anatómicamente en la circunvolución frontal superior, limitada a nivel posterior por el surco precentral y el córtex motor primario, y a nivel medial por el surco del cíngulo, con un límite anterior aun hoy en día mal definido ${ }^{22,24,27,33}$. Se le atribuyen funciones de inicio de movimiento, actuando como conexión entre el sistema límbico y el aparato motor ejecutivo, de modo que actúa traduciendo el motivo de la intención a la acción y ejerciendo el control sobre el córtex motor primario ${ }^{39}$. El AMS participa por tanto en la preparación, iniciación y monitorización de movimientos complejos, así como en la recuperación y repetición de tareas complejas aprendidas previamente ${ }^{24,27}$. Otra función atribuible al AMS es su participación en tareas de lenguaje. El AMS participa en la iniciación y ritmo del lenguaje, en el control de la articulación y de la fonación ${ }^{7,24,27,39}$.

La cirugía en el AMS o en las proximidades de la misma, puede producir un conjunto de signos y síntomas deficitarios característicos que se engloban en el llamado síndrome del $\mathrm{AMS}^{7,9,10,17,22,31,39}$.

El síndrome motor completo se manifiesta como una hemiplejia contralateral en los primeros días postoperatorios. En los siguientes días la recuperación es progresiva, observándose un enlentecimiento en la respuesta motora desde su inicio voluntario hasta su ejecución definitiva ${ }^{7,13-15,17,22,24,31}$. El movimiento de las extremidades puede resultar normal durante acciones automáticas, pero no durante su intento de uso voluntario ${ }^{22}$. Puede acompañarse de hemiataxia, hemiapraxia, heminegligencia, hiperreflexia e hipertonicidad. La recuperación del movimiento acostumbra a ser completa en 11 días. Los movimientos finos pueden tardar de 2 a 6 semanas en recuperar. Las tareas complejas o que requieran especial destreza, así como los movimientos a gran velocidad o sinergia bimanual, puede quedar como déficit permanente $2,8,9,11,12,17-19,22,24,27,31,39$.

El síndrome deficitario de lenguaje aparece en lesiones del AMS en el hemisferio dominante y se caracteriza en su forma extrema por mutismo. Este defecto acostumbra a recuperarse paulatinamente, dando paso a una disfasia transcortical motora con disfasia severa de predominio

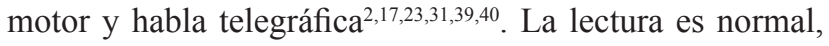
la repetición se altera poco y puede haber ecolalia y perseveración de palabras o pequeñas frases durante 1-2 semanas $1,3,17,21,31,39$. También se describen en la literatura déficits permanentes de lenguaje, que incluyen alteraciones en fluencia y aprendizaje verbal, alteraciones en la comprensión de lenguaje de gran complejidad o expresado a gran velocidad y dificultad en el lenguaje bajo stress ${ }^{9,12,15,17-}$ $19,23,24,27,30,31,39$

La presencia transitoria del síndrome se atribuye a otra característica importante del AMS: su bilateralidad. A parte de sus múltiples y complejas conexiones con los ganglios basales, cíngulo, cerebelo, tálamo, médula espinal, el AMS de cada hemisferio mantiene conexión bilateral con el córtex motor y con el AMS contralateral $4,13,17,20,24,27,28,35,36$. Existe también cierta dominancia en el $\mathrm{AMS}^{29}$, de manera que la manipulación del hemisferio izquierdo, hemisferio habitualmente dominante, supondría mayor déficit o mayor duración del mismo.

La recuperación relativamente rápida de estos síntomas en gran parte de los pacientes con lesiones del AMS intervenidas, nos hace cuestionar la necesidad o no de identificar y preservar este área ${ }^{17,22,31}$. En las series quirúrgicas se ha podido observar que muchos síntomas, motores y de lenguaje por afectación del hemisferio dominante, pueden ser molestos e incluso invalidantes dependiendo del status o profesión del paciente $\mathrm{e}^{2,6,7,24,39}$. Otras publicaciones muestran la persistencia del síndrome del AMS después de accidentes vasculares cerebrales isquémicos en esta zona anatómica ${ }^{1,5,21,23,32}$. Estos hechos obligan a plantearse nue- 
vamente la creencia de que la reversibilidad del síndrome AMS, explicado por la bilateralidad del AMS, pueda generalizarse a todos los pacientes con lesión en este área.

Existen numerosos estudios mediante resonancia magnética funcional (RMf) que utilizan distintas tareas para activar el AMS. La tarea motora más utilizada, por considerarse más específica, es la contraposición de dedos con el pulgar o tapping ${ }^{16,22,33,34,38}$. La utilización de tareas similares a las utilizadas para identificar el AMS mediante RMf podrían realizarse en el quirófano, solicitando al paciente que realice una tarea motora específica y después bloquear la ejecución de esta tarea mediante estimulación eléctrica cortical. Para este efecto es necesario que el paciente esté despierto y colaborador, lo que obliga a realizar estos procedimientos con anestesia local. No existen actualmente estudios intraoperatorios con estimulación cortical directa para la identificación específica del AMS mediante bloqueo de una tarea específica. En nuestro centro intervenimos la mayoría de lesiones ubicadas alrededor de la circunvolución motora bajo anestesia local siguiendo las indicaciones de algunos autores ${ }^{37}$. Este hecho nos ha conducido a intentar identificar el AMS en los pacientes con lesiones promotoras utilizando un método sencillo, que no añada morbilidad ni alargue en exceso el tiempo de la intervención.

El objetivo principal del presente estudio prospectivo es conseguir la identificación intraoperatoria de la función motora del AMS mediante estimulación eléctrica cortical y bloqueo de una tarea motora intraoperatoria en pacientes con tumores cerebrales ubicados en este área. El objetivo secundario es valorar el pronóstico funcional tras la preservación de este área durante el tratamiento quirúrgico.

\section{Pacientes y método}

Desde enero del 2004 hasta enero del 2007 identificamos 15 pacientes con lesión pre-rolándica. En todos ellos se practicó un estudio RMf específico para identificación del córtex motor primario y del AMS. Para ello se ha escogido una tarea de oposición entre el dedo pulgar y dedos alternos (secuencia $2^{\circ}-4^{\circ}-3^{\circ}-5^{\circ}$ ) en la mano contralateral al hemisferio donde se encuentra la lesión ${ }^{22}$.

La craneotomía se realizó con el paciente despierto y anestesia local (solución de Svedocaina $0.25 \%$ 60cc, Lidocaina $2 \% 30 \mathrm{cc}$ y Bicarbonato 1 molar $4 \mathrm{cc}$ ) en todos los casos. Una vez realizada la craneotomía, se procede a la identificación anatómica con la ayuda del neuronavegador y se confirma mediante estimulación eléctrica cortical utilizando el estimulador de Ojemann OCS (Ojemann Cortical Stimulator, OCS Radionics, Burlington, Massachusets, USA). Se inicia la identificación del córtex motor, marcando mediante etiquetas plastificadas de color rojo numeradas las áreas correspondientes a cada movimiento

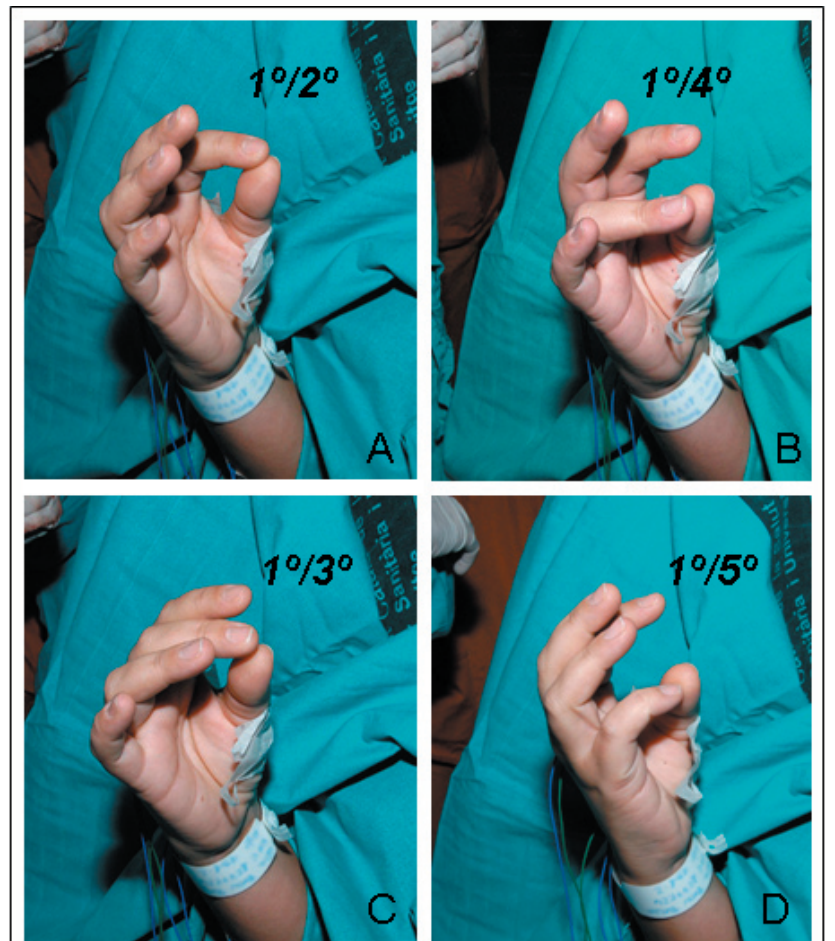

Figura 1. Tarea motora intraoperatoria de oposición de dedos (tapping). A. Inicio de la tarea con la oposición del segundo dedo con el pulgar. B. Continuación con la oposición del cuarto dedo con el pulgar. C. Oposición del tercer dedo con el pulgar. D. Oposición del quinto dedo con el pulgar.

obtenido. Proseguimos con la identificación del córtex sensitivo, marcando con etiquetas azules numeradas las áreas correspondientes a las sensaciones obtenidas. Posteriormente iniciamos la identificación funcional del AMS. Para ello pedimos al paciente que realice durante la estimulación la misma tarea de oposición de dedos descrita durante la realización de la RMf. Identificamos como áreas funcionales correspondientes al AMS aquellos puntos donde se bloquea dicha tarea. La estimulación cortical empieza a la misma intensidad que precisamos durante la estimulación del córtex sensitivomotor, y se incrementa en intervalos de $0.5 \mathrm{~mA}$ cada vez que no se obtiene respuesta. Estas áreas se marcan con etiquetas amarillas numeradas. Cada una de las etiquetas responde a una función determinada que se anota en la hoja para la recogida de datos correspondiente. Una vez identificadas todas las áreas se procede a la resección de la lesión preservando las zonas marcadas como funcionales. En caso de encontrar infiltración tumoral en zonas marcadas como funcionales en el AMS, se resecarán junto al tumor obviando su función.

Para el análisis estadístico se procedió al estudio de la distribución de cada una de las variables por medio del test de Kolmogorov- Smirnov, que se confirmó por medio 


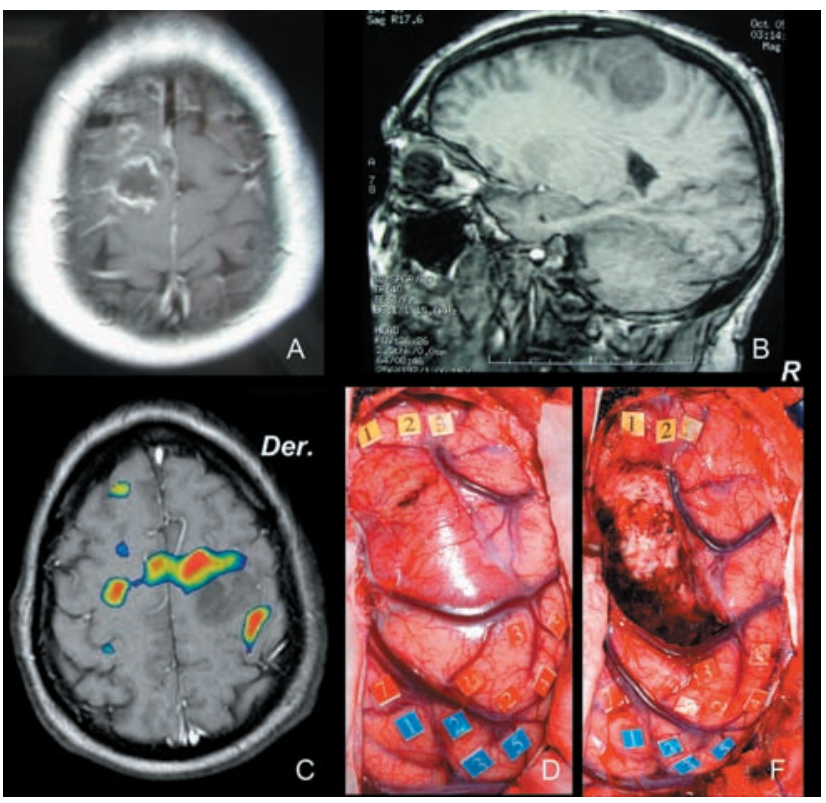

Figura 2. Caso clínico perteneciente a un varón de 65 años con clínica de hemiparesia braquio crural izquierda. A. RM cerebral en secuencia T1 con gadolinio que muestra una lesión intraaxial premotora con captación periférica y centro necrótico. B. Secuencia T1 sagital sin contraste. C. $R M f$ que muestra una extensa región funcional para el área motora suplementaria derecha anterior a la lesión y una región funcional posterior a la lesión correspondiente al área motora primaria. D. Mapping intraoperatorio sensitivo (etiquetas azules), motor (etiquetas rojas) y motor suplementario (etiquetas amarillas), obtenidos mediante estimulación eléctrica cortical en el paciente despierto, con buena correlación con los hallazgos en la RMf (C).

de las gráficas P-P y Q-Q. Se observó que la mayoría no seguían una distribución normal $\mathrm{y}$, dado que el número de pacientes fue reducido $(\mathrm{n}=15)$, se emplearon pruebas no paramétricas para realizar las comparaciones. Para determinar la asociación entre las variables independientes binarias (pronóstico neurológico a corto y largo plazo) y variables cuantitativas, se empleó el test de la U de MannWhitney. La asociación entre las variables independientes binarias (pronóstico neurológico a corto y largo plazo) y las variables cualitativas se determinó utilizando el test exacto de Fisher. En todos los casos se aceptó un nivel de significación del 5\% $(\mathrm{p}<0,05)$. Para el análisis estadístico se utilizó el programa SPSS versión 15.0.

\section{Resultados}

Los síntomas preoperatorios fueron déficit motor en 7 pacientes ( 5 hemiparesia, 2 monoparesia crural), crisis epilépticas en 5 pacientes, y 3 eran pacientes asintomáticos con recidiva de lesión previamente intervenida (2 astrocitomas anaplásicos y 1 oligodendroglioma II) diagnosticada en controles ordinarios mediante RM. El estudio anatomopatológico de todos los tumores resecados se especifican en la Tabla 1. Diez se ubicaban en hemisferio derecho y cinco en el izquierdo. El tamaño promedio fue de $4.06 \mathrm{~cm}$. de diámetro máximo.

La intensidad del estímulo promedio para la identificación del córtex motor primario fue de $2.5+-1 \mathrm{~mA}$. La misma intensidad se precisó para la identificación del córtex sensitivo. Con esta intensidad de estímulo se consigue identificar el córtex sensitivomotor en todos los pacientes estudiados. Para la identificación intraoperatoria del área motora suplementaria, se precisó intensificar el estímulo hasta $3+-1 \mathrm{~mA}$ de promedio. Con esta intensidad de estímulo se consigue el bloqueo de la tarea motora de dedos alternos en todos los pacientes estudiados.

En todos los pacientes existió una correcta correlación entre la RMf y los hallazgos de la estimulación cortical intraoperatoria. La resección fue macroscópicamente completa en 13 pacientes (86.6\%) y subtotal en 2 (13.3\%). En dos pacientes se identifica intraoperatoriamente una infiltración del córtex motor y de la vía motora. En estos dos pacientes la resección final fue subtotal, dejando un resto de lesión en el córtex motor marcado como funcional. La función encontrada en el AMS se ha podido preservar en 14/15 pacientes $(93,3 \%)$. Sólo en una paciente se resecó parcialmente el AMS funcional junto a la lesión por infiltración de la misma (6.6\%). En $7 / 15$ pacientes $(46.6 \%)$ el mapping cortical fue indispensable para identificar y preservar el córtex motor, en 4/7 por fallo en la correlación de la venografía superficial con los surcos y circunvoluciones reales y en $3 / 7$ por alteración de la anatomía normal al tratarse de pacientes reintervenidos.

En el postoperatorio inmediato $8 / 15$ pacientes $(53.3 \%)$ presentaron empeoramiento de los déficits o aparición de nuevos síntomas deficitarios, transitorios o definitivos. Siete de estos pacientes con déficit ( $46 \%$ del total) presentaron mayor o menor grado de síndrome del área motora suplementaria claramente distinguible. De los 9 pacientes con lesiones derechas, 1 paciente presentó un síndrome del AMS leve y 1 paciente completo. De los 6 pacientes con lesiones izquierdas, 1 paciente presentó un síndrome del AMS leve y 4 pacientes completo. Cinco pacientes (33.3\%) no mostraron cambios respecto a su exploración preoperatoria y 2 pacientes mejoraron. De los pacientes con lesiones izquierdas, $5 / 6$ presentaron disfasia transitoria y déficit motor postquirúrgico. De los pacientes con lesión derecha 7/9 presentaron déficit motor (primario o AMS) y ninguno de ellos déficit de lenguaje.

A los 6 meses de la intervención $5 / 15$ pacientes $(33.3 \%)$ se encuentran asintomáticos y $10 / 15$ pacientes presentan déficits permanentes. En este último grupo, 5/10 pacientes 


\begin{tabular}{|c|c|c|c|c|c|c|c|c|c|c|c|c|c|c|c|}
\hline$\hat{\underline{z}}$ & 8 & \& & $\infty$ & $\stackrel{8}{1}$ & 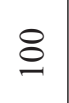 & 8 & \& & 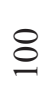 & 8 & \& & $\triangleright$ & \& & 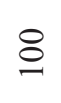 & in & 8 \\
\hline 总言 & $\infty$ & $\infty$ & 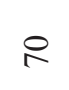 & 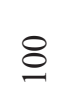 & $\infty$ & 8 & 8 & 8 & 8 & $R$ & 8 & 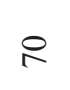 & 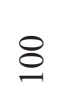 & in & 8 \\
\hline$\frac{n}{\underline{z}}$ & 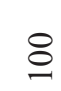 & $R$ & $\infty$ & \& & $\infty$ & 8 & \& & $\triangleright$ & 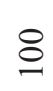 & 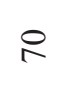 & $\infty$ & $尺$ & 8 & 8 & 8 \\
\hline
\end{tabular}

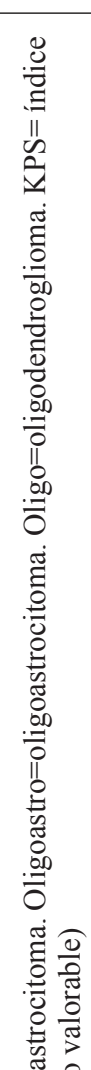

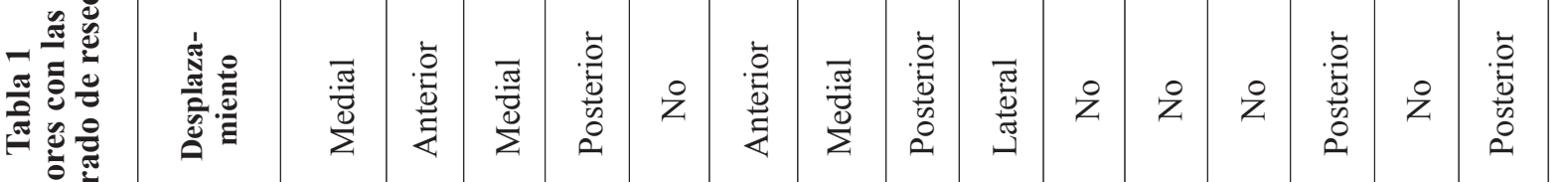

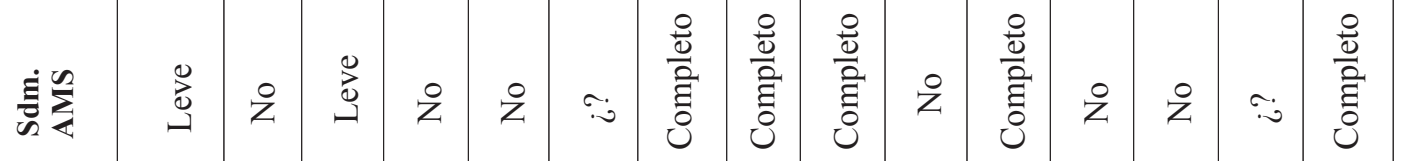

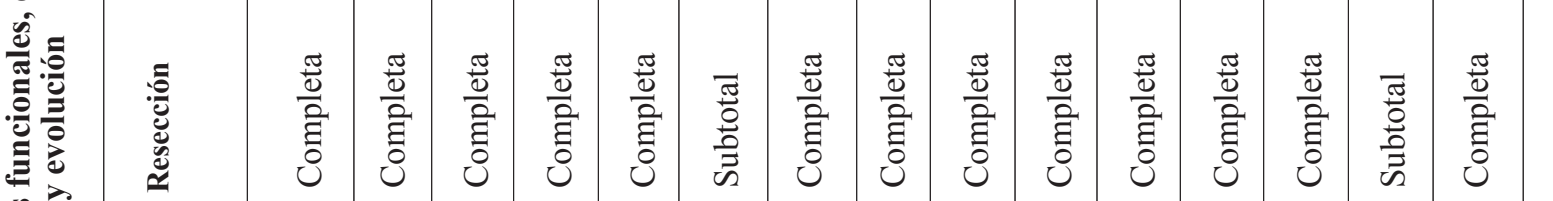

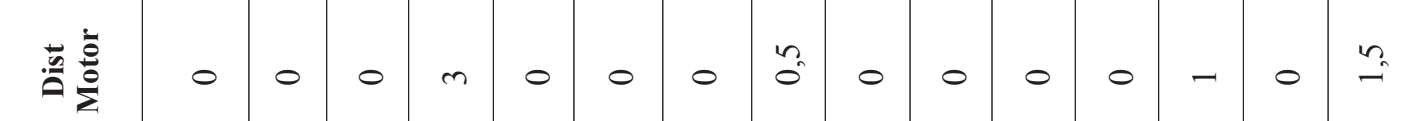

焉蒙

\begin{tabular}{|c|c|c|c|c|c|c|c|c|c|c|c|c|c|c|c|}
\hline 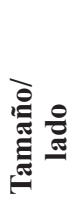 & : & $\begin{array}{l}\text { हैं } \\
\text { है } \\
\text { ñ }\end{array}$ & 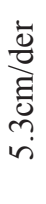 & 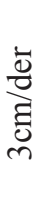 & 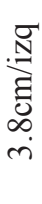 & 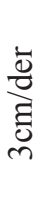 & 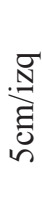 & 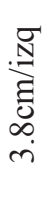 & 胥 & 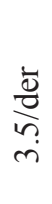 & 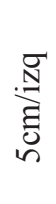 & $\frac{\bar{d}}{\frac{\pi}{n}}$ & 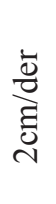 & 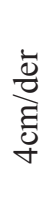 & $\begin{array}{l}\overrightarrow{0} \\
\text { हे } \\
\text { ñ }\end{array}$ \\
\hline 离 & 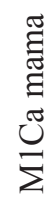 & $\begin{array}{l}\tilde{\Xi} \\
\text { ज्ञ } \\
\text { Uू } \\
\Sigma\end{array}$ & $\sum_{0}^{\infty}$ & $\sum_{0}^{\infty}$ & $\begin{array}{l}\text { 岁 } \\
\bar{\Sigma}\end{array}$ & $\sum_{0}^{\infty}$ & 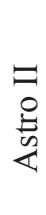 & $\begin{array}{l}= \\
0 \\
0 \\
0 \\
0 \\
0 \\
.00 \\
0\end{array}$ & $\begin{array}{l}\exists \\
.00 \\
.00 \\
0\end{array}$ & 宓 & 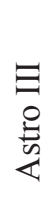 & $\begin{array}{l}\text { 目 } \\
\text { 章 } \\
\end{array}$ & $\sum_{0}$ & $\sum_{0}^{\infty}$ & 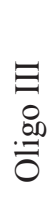 \\
\hline 芫 & $\underset{\infty}{\Xi}$ & $\begin{array}{l}\Xi \\
\text { in }\end{array}$ & $\begin{array}{l}\Xi \\
\text { ปे }\end{array}$ & $\begin{array}{l}\vec{\infty} \\
\stackrel{\infty}{+}\end{array}$ & ì & 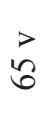 & ఏ్ & in & 只 & $\vec{n}$ & 8 & $\begin{array}{l}\vec{\infty} \\
\stackrel{\infty}{+}\end{array}$ & $\begin{array}{l}\Xi \\
\stackrel{n}{n}\end{array}$ & $\vec{n}$ & $\begin{array}{l}\vec{n} \\
i n\end{array}$ \\
\hline & - & $N$ & $m$ & $\nabla$ & in & 6 & $r$ & $\infty$ & $a$ & 으 & $=$ & $\simeq$ & 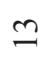 & $\Xi$ & $n$ \\
\hline
\end{tabular}


$(33.3 \%)$ muestran un déficit muy leve que no interfiere en su vida normal (2 hemiparesia 5-/5, 2 monoparesia crural 5-/5, 1 hipostesia crural leve). En los otros 5/10 pacientes $(33.3 \%)$ los déficits permanentes interfieren con la vida del paciente: dos pacientes presentan hemiparesia severa 3/5 (igual estado preoperatorio que no mejora), uno afasia de expresión y otros dos (13.3\%) presentan un síndrome del AMS izquierda permanente caracterizado por enlentecimiento en la iniciación del movimiento y dificultad para movimientos finos asociados a trastorno del lenguaje con enlentecimiento del ritmo y disfasia leve. El paciente con afasia de expresión se relaciona, por su momento de aparición, con la manipulación intraoperatoria del límite inferior de la lesión colindante con el área de Broca anatómica. En los 2 pacientes con hemiparesia severa se objetivó intraoperatoriamente una infiltración tumoral del córtex motor primario en el primero y de la substancia blanca subcortical correspondiente a la vía motora en el segundo. El KPS (índice de Karnofsky) preoperatorio promedio es de 82 , el postoperatorio inmediato de 70 y a los 6 meses de 87 . Los 2 pacientes con KPS preoperatorio $<70$ no recuperan sus déficits motores y se trata de los mismos pacientes con lesión infiltrante de la vía motora. El 100\% de los pacientes con déficit motor severo (KPS $<70$ ) antes de la cirugía, tuvieron déficit motor severo persistente tras la cirugía, mientras que sólo en 1 de los 13 pacientes $(7.7 \%)$ sin déficit motor severo antes de la cirugía, tuvo déficit neurológico severo persistente tras la cirugía. Las diferencias entre estas 2 proporciones resultaron ser estadísticamente significativas $(\mathrm{p}=0,03)$.

Para conocer la distancia del tumor a las áreas funcionales, se ha medido la distancia más corta desde el tumor a las etiquetas corticales (Tabla 1$)$. En $11 / 15$ pacientes $(73,3 \%)$ la distancia del tumor al córtex motor fue cero, es decir, que el tumor se encontraba en íntimo contacto con las zonas marcadas como funcionales, sin interposición de córtex cerebral entre lesión y área funcional. En 9/15 pacientes $(60 \%)$ la distancia entre el tumor y el AMS fue cero, también. Existe desplazamiento de la función del AMS en $10 / 15$ pacientes $(66.6 \%)$. Para describir cualitativamente el tipo de desplazamiento de la función del AMS por el tumor, se ha valorado la dirección del desplazamiento anterior (2 pacientes), posterior (4 pacientes), medial (3 pacientes) y lateral (1 paciente). En cuatro pacientes no existe desplazamiento en la función del AMS.

En los grupos de pacientes con y sin empeoramiento neurológico a largo plazo tras la cirugía, se compararon las siguientes variables: edad, diámetro máximo del tumor, distancia entre el tumor y el AMS, distancia entre el tumor y el córtex motor primario, tipo de desplazamiento de la función del AMS respecto al tumor (anterior, posterior o lateral), tipo histológico, calidad de la resección tumoral (completa o no completa) y aparición de síndrome de AMS en el postoperatorio. Ninguna de estas comparaciones fue estadísticamente significativa $(p>0.05)$. Las mismas variables se compararon en los grupos de pacientes con y sin empeoramiento neurológico a corto plazo tras la cirugía. Ninguna de estas comparaciones fue estadísticamente significativa $(\mathrm{p}>0.05)$.

\section{Discusión}

\section{Estimulación cortical en el paciente despierto}

Hemos escogido intervenir los pacientes con tumores adyacentes al córtex motor con el paciente despierto y anestesia local siguiendo el estudio de Vitaz et al $^{37}$. Según este autor, el paciente despierto mejora las posibilidades de identificación del área motora, disminuye la incidencia de crisis epilépticas. El paciente despierto también nos ofrece la posibilidad de identificar, a parte del córtex motor, el córtex sensitivo y del AMS. En esta serie, el mapeo cortical sensitivomotor y del AMS en el paciente despierto ha ayudado en la orientación anatómica y su relación con el tumor a partir de las funciones identificadas, y este hecho queda patente en dos circunstancias distintas. Por un lado, en las diferencias anatómicas individuales y la confusión que genera la anatomía venosa superficial, que a menudo no se corresponde con el recorrido de las circunvoluciones. El neuronavegador no es suficiente ayuda y precisamos del mapeo cortical para asegurar una correcta localización funcional de, al menos, el córtex motor, íntimamente relacionado con el aspecto posterior y posteromedial del AMS. En el $46.6 \%$ de pacientes el mapping cortical contribuyó a la orientación anatómica e influyó en la resección. Por otro lado, cabe citar como caso especial a los 3 pacientes con recidiva de lesiones previamente tratadas con cirugía y radioterapia. La apertura dural en estos pacientes fue difícil debido a la gran adherencia de la duramadre a la superficie cerebral, resultando un área de decorticación perilesional que imposibilitaba cualquier identificación anatómica. El mapeo cortical fue imprescindible para delimitar y preservar las distintas zonas funcionales en íntima relación con la lesión. No se ha encontrado relación estadística entre tratamiento quirúrgico, incluida la estimulación cortical, y la aparición de empeoramiento postquirúrgico a corto $\mathrm{y}$ largo plazo, por lo que podemos deducir que el mapeo del AMS en el paciente despierto no añade morbilidad al procedimiento quirúrgico.

\section{Tarea intraoperatoria}

Penfield y Welch en $1951^{26}$ describieron el AMS como un área ubicada en la parte anterior del extremo superior del córtex motor, incluyendo la porción de córtex premotor correspondiente a la representación cortical motora 
para la pierna contralateral, con una función de movimiento distinta a la del córtex motor primario. Como respuestas más destacadas en la estimulación de este área, describieron movimientos en el brazo contralateral, aunque también en la pierna, parada o enlentecimiento durante la ejecución de una acción voluntaria ya sea motora o de lenguaje, y vocalización de sonidos. Así mismo pudieron observar que la estimulación utilizando voltajes elevados puede inducir crisis adversivas. Existe acuerdo en que la función del AMS está implicada en convertir la intención en ejecución de un movimiento o del habla, que su lesión impide los movimientos voluntarios y que sus secuelas permanentes alteran los movimientos finos o que necesiten coordinación bimanual predominantemente contralaterales a la lesión, y también la iniciación del habla o el lenguaje bajo stress. En nuestra serie hemos utilizado una tarea motora compleja que consiste en la oposición de dedos alternos en la mano contralateral a la lesión (tapping). Esta tarea permite el estudio previo con RMf y su repetición exacta en quirófano, siendo bloqueada mediante estimulación eléctrica. La realización de esta tarea requiere al paciente despierto y colaborador durante la intervención, por lo que resulta imprescindible realizar esta parte del procedimiento bajo anestesia local. El bloqueo de función se obtiene con facilidad, requiriendo menor voltaje que la consecución de movimiento y por tanto disminuye el riesgo de crisis intraoperatorias. El uso de esta tarea motora compleja ha permitido la identificación del AMS en el $100 \%$ de los pacientes intervenidos.

\section{Relación con el córtex y vía motora primaria}

La proximidad del AMS al córtex motor primario, puede confundir el diagnóstico de procedencia de la causa del déficit motor postquirúrgico ${ }^{2}$. Algunos autores refieren el déficit motor con origen en la lesión del AMS como "rápidamente reversible", convirtiendo esta característica en definición ${ }^{22,27,39}$. En nuestra experiencia, la estimulación eléctrica motora y del AMS, así como su manipulación quirúrgica, pueden inducir por sí mismos un déficit transitorio también de rápida reversibilidad. Hemos utilizado sistemáticamente la estimulación eléctrica cortical en todos los pacientes de esta serie, por lo que la reversibilidad clínica de los déficits no nos resulta orientativa en cuando al origen de los mismos, siendo necesario buscar otros datos clínicos. El déficit motor perteneciente al síndrome AMS característicamente presenta movimientos reflejos espontáneos con ausencia de movimiento voluntario o a la orden ${ }^{17}$. En nuestra serie este hecho ha sido constante en todos los pacientes. En tres casos se ha observado también una hipertonicidad muy marcada en el hemicuerpo afectado así como heminegligencia ${ }^{7,15,27,31}$. Cuando el síndrome es mixto y grave, el déficit motor primario enmascara el síndrome del
AMS. Este hecho se ha podido observar en 3 pacientes. En dos de ellos la lesión era derecha y el déficit preoperatorio ya era muy grave, sin recuperación en el postoperatorio. El tercer paciente, con una lesión izquierda que ocupaba gran parte de la circunvolución motora, presentó un síndrome completo postoperatorio, con mejoría rápida del déficit motor pero con persistencia del trastorno de lenguaje, neuropsicológicamente descrito como una afasia de expresión pura, posiblemente por lesión intraoperatoria del área de Broca anatómica colindante con el límite antero-inferior de la lesión.

Como hemos mencionado, la cercanía del AMS al córtex y vía motora aumenta obviamente el riesgo de lesionar estas estructuras durante el acto quirúrgico ${ }^{27}$. No debemos olvidar, por la frecuente recuperación del síndrome del AMS, que el principal objetivo funcional en la resección de lesiones en este área debe seguir siendo la preservación de la función motora primaria. Cuando la distancia entre el tumor y el córtex motor primario es inferior a $0.5 \mathrm{~cm}$, se relaciona con aparición de déficit motor primario o síndrome de AMS en la resección de tumores u otras lesiones $^{22,27}$. Este dato parece correlacionarse también con los hallazgos en nuestra serie, aunque el estudio estadístico no resulte significativo, probablemente por tratarse de una muestra pequeña; de los 11 pacientes con lesiones ubicadas a una distancia inferior a $0.5 \mathrm{~cm}$ del córtex motor, 6 pacientes empeoran o añaden síntomas en el postoperatorio, y en 5 de estos 6 pacientes la recuperación es buena a los 6 meses (KPS 100 en 2, 90 en 2 y 80 en 1). En 4 pacientes la distancia al córtex motor fue superior a $0.5 \mathrm{~cm}, 2$ de estos pacientes empeoran levemente en el postoperatorio y su recuperación a los 6 meses es excelente en todos ellos (KPS 100 en 3 y 90 en 1). En 2 de nuestros pacientes quedaron déficits motores permanentes claramente pertenecientes a la lesión del córtex motor primario, y en ambos pacientes se objetivó intraoperatoriamente de forma muy evidente la infiltración cortical y subcortical de la circunvolución rolándica. Estos 2 pacientes presentaban un déficit motor grave preoperatorio y KPS $<70$. Ninguno de ellos recuperó su déficit motor. El KPS $<70$ preoperatorio en pacientes con lesiones en el AMS se relaciona con la afectación directa de la vía motora y es significativamente un indicador de mal pronóstico funcional y de escasa capacidad de recuperación, o nula en el presente estudio.

\section{Secuelas permanentes en relación al AMS}

Penfield ${ }^{25}$ ya advirtió de la posibilidad de aparición de secuelas permanentes como consecuencia de la ablación quirúrgica de este área, y lo ejemplificaba en pacientes con lesiones derechas tras cirugía de la epilepsia. Después de un año de seguimiento, describe persistencia de lentitud de movimientos cuando son repetidos o alternantes en 
Tabla 2

Evolución neurológica respecto al síndrome del AMS en las principales series de pacientes con lesiones pre-motoras

\begin{tabular}{|c|c|c|c|c|c|c|c|c|}
\hline Autor & Año & $\begin{array}{c}\text { Tiempo } \\
\text { seguimiento }\end{array}$ & Pacientes & Sin déficit & \multicolumn{2}{|c|}{$\begin{array}{l}\text { Déficits } \\
\text { transitorios }\end{array}$} & \multicolumn{2}{|c|}{$\begin{array}{c}\text { Déficits } \\
\text { permanentes }\end{array}$} \\
\hline Laplane et $\mathrm{al}^{17}$. & 1977 & - & 3 & 0 & $3 / 3$ & $100 \%$ & $3 / 3$ & $100 \%$ \\
\hline Rostomily et a ${ }^{31}$. & 1991 & 12 meses & 6 & 0 & $6 / 6$ & $100 \%$ & 0 & $0 \%$ \\
\hline Zetner et al ${ }^{39}$. & 1996 & 6-48 meses & 28 & 3 & $22 / 28$ & $78.5 \%$ & $3 / 28$ & $10.7 \%$ \\
\hline Bannur \& Rajshekhar ${ }^{2}$ & 2000 & 8-38 meses & 6 & 0 & $4 / 6$ & $66.6 \%$ & $2 / 6$ & $33.3 \%$ \\
\hline Krainik et al ${ }^{15}$. & 2001 & 12 meses & 23 & 12 & $6 / 23$ & $26 \%$ & $5 / 23$ & $21.7 \%$ \\
\hline Peraud et $\mathrm{a}^{27}$. (?) & 2002 & 12 meses & 24 & 4 & $16 / 24$ & $66.6 \%$ & $7 / 27$ & $29.1 \%$ \\
\hline Fontaine et $\mathrm{al}^{7}$. & 2002 & 3 meses & 11 & 0 & $9 / 11$ & $81.8 \%$ & $2 / 11$ & $18.8 \%$ \\
\hline Nelson et $\mathrm{al}^{22}$. & 2002 & - & 12 & 9 & $3 / 12$ & $25 \%$ & 0 & $0 \%$ \\
\hline Duffau et $\mathrm{al}^{6}$. & 2003 & 12 meses & 25 & 0 & $25 / 25$ & $100 \%$ & 0 & $0 \%$ \\
\hline Gabarrós et al.* & 2007 & 12 meses & 15 & 5 & $5 / 15$ & $33.3 \%$ & $2 / 15$ & $13.3 \%$ \\
\hline
\end{tabular}

$(?)$ = Posible lesión del córtex motor no especificada. *Lesión motora/lenguaje sin relación con el AMS en 3 pacientes).

el hemicuerpo contralateral y, en algún caso, ipsilateral también. Específicamente menciona la dificultad en un paciente para escribir a máquina con la mano contralateral (izquierda), fenómeno que se repite con los pacientes de nuestra serie con déficits permanentes. Desde la descripción del síndrome del AMS por Laplane en $1977^{17}$, se han registrado numerosos casos de persistencia de síntomas postquirúrgicos $^{2,7,27,39}$. En nuestra serie, en el 33.3\% de pacientes el síndrome del AMS fue transitorio, desapareciendo totalmente en un tiempo máximo de 6 meses. En el $13.3 \%$ de los casos, a pesar de una recuperación de la mayor parte de los síntomas del síndrome, quedan secuelas permanentes de tipo motor y de lenguaje en las lesiones del hemisferio dominante. Uno de estos dos pacientes es el único caso en que se resecó parte del AMS funcional por infiltración tumoral, lo que explicaría la aparición de un déficit permanente, aunque la manipulación quirúrgica en la proximidad de áreas funcionales (shock quirúrgico), y el edema resultante, puede ser suficiente para explicar los déficits postquirúrgicos que posteriormente se recuperan ${ }^{31}$. En el otro paciente, el motivo más probable es la lesión quirúrgica de la vía que conduce información desde el AMS.

Hemos podido observar en nuestra serie que los tumores que tienen contacto directo con el AMS, acostumbran a desplazar (66.6\%) más que a invadir (6.6\%) este área funcional. El desplazamiento de la función facilita la resección lesional y quizás disminuya el riesgo de dejar secuelas permanentes. Asimismo también, los tumores metastáticos, que habitualmente presentan un plano de resección no infiltrante y más accesible, tienen mejor pronóstico funcional $^{31}$. Estos hallazgos nos invitan a pensar que al identificar intraoperatoriamente el AMS durante la resección de tumores colindantes, nos permitiría limitar la resección respetando este área funcional y disminuyendo la aparición de déficits neurológicos transitorios y permanentes. Hemos intentado buscar alguna contestación a esta hipótesis revisando series en la literatura que describen la extirpación de lesiones ubicadas en córtex premotor sin identificación funcional del AMS, y se han comparado las secuelas de estos pacientes con los de nuestra serie.

Zentner et al. en $1996^{39}$, publica una de las series más largas y mejor documentadas en cirugía del AMS. De 28 pacientes intervenidos que incluyen lesiones tumorales y no tumorales, 22 pacientes presentan déficits transitorios y 3 pacientes presentan déficits permanentes en relación al síndrome del AMS tales como imposibilidad de realizar tareas motoras complejas, especialmente a alta velocidad, precisión o sinergia bimanual. Fontaine et al. en el $2002^{7}$, en una serie de 11 pacientes con gliomas, 2 presentan déficits permanentes consistentes en dificultades en la iniciación 
del movimiento o del habla. En 7/11 pacientes la lesión era izquierda y practicaron mapeo de lenguaje en paciente despierto utilizando tareas intraoperatorias de nominación y contar números, induciendo un speech arrest o parada del habla en las áreas de lenguaje. Estas áreas eran respetadas, deteniendo la resección a $7 \mathrm{~mm}$ de las zonas marcadas para lenguaje, pero algunas de estas áreas hubieran podido estar en relación con el AMS izquierda desplazada que responde al mismo tipo de estímulo y con la misma respuesta, speech arrest, según describió Penfield ${ }^{25,26}$, y según la experiencia de nuestro grupo. Para poder diferenciar el área propia del lenguaje del AMS se necesitaría una tarea más específica para estudiar la función del lenguaje en el AMS, como una tarea de generación de verbos que implica funciones de selección, preparación y ejecución propias del AMS. Hipotéticamente, de no haber respetado algunas de estas áreas pertenecientes al AMS, quizás el número de pacientes con lesión permanente hubiera sido mayor en esta serie. Laplane ${ }^{17}$, describe déficits permanentes en $3 / 3$ pacientes. Rostomily ${ }^{31}$, en su estudio sobre 6 pacientes y Nelson ${ }^{22}$, en su estudio sobre 12 pacientes, no muestran secuelas por lesión del AMS a largo plazo, aunque en esta última serie, en la mayoría de pacientes la distancia del tumor al AMS es superior a $1 \mathrm{~cm}$. La serie de Peraud $^{27}$, de 24 pacientes intervenidos de glioma de bajo grado, cuenta $7 / 21$ pacientes con lesiones permanentes, pero no establece con claridad si el tipo de lesión pertenece a síntomas motores puros o del AMS. Duffau 6 , mediante estimulación eléctrica cortical intraoperatoria con el paciente despierto, identifica y preserva las áreas de lenguaje encontradas en una serie de 25 pacientes con glioma de bajo grado premotor izquierdo; todos los pacientes presentan déficit posquirúrgico inmediato, pero hay que destacar que ninguno de ellos presenta déficits permanentes en relación al AMS. En el conjunto de la literatura, teniendo en cuenta las series donde no se identifica el AMS con el paciente despierto, la tasa de lesión transitoria es del $61.6 \%$ y la tasa de lesión permanente es del 19.46\%. En nuestra serie, preservando el AMS en 14/15 pacientes, la tasa de lesión transitoria es del $33.3 \%$ y permanente en el $13.3 \%$. Según estos datos, la identificación y preservación del AMS ayudaría a disminuir claramente la incidencia del síndrome del AMS en el postoperatorio, y disminuiría también, aunque no de forma tan evidente, la tasa de lesión permanente. Si tenemos sólo en cuenta los 14 pacientes en que se preservó funcionalmente el AMS, la tasa de lesión permanente resulta entonces del $6.6 \%$, significativamente inferior a la de la literatura. Para confirmar estos datos serían necesarios más estudios con un numero mayor de pacientes, pero parece obvio de la identificación del AMS en el paciente despierto y su preservación, siempre que sea posible, ayuda a disminuir la tasa de lesión permanente.

\section{Conclusiones}

La estimulación cortical intraoperatoria resulta útil para la identificación del AMS. La preservación del AMS, una vez identificada, disminuye la aparición de síntomas postquirúrgicos y la persistencia del síndrome del AMS. Cuando el AMS se encuentra infiltrada por tumor, su resección quirúrgica puede causar déficits permanentes, especialmente en el hemisferio dominante. El déficit motor severo preoperatorio se asocia con mal pronóstico funcional.

\section{Bibliografía}

1. Alexander, M.P., Schmitt, M.A.: The aphasia syndrome of stroke in the left anterior cerebral artery territory. Arch Neurol 1980; 37: 97-100.

2. Bannur, U., Rajshekhar, V.: Post operative supplementary motor area syndrome: clinical features and outcome. $\mathrm{Br} \mathrm{J}$ Neurosurg 2000; 14: 204-210.

3. Berthier, M.L, Starkstein, S.E, Leiguarda, R., et al.: Transcortical aphasia. Importance of the nonspeech dominant hemisphere in language repetition. Brain 1991; 114: 14091427.

4. Brinkman, J., Porter, R.: Supplementary motor area of the monkey: activity of neurones during performance of a learned motor task. J Physiol 1978; 74: 313-316.

5. Dick, J.P., Benecke, R., Rothwell, J.C., Day, B.L., Marsden, C.D.: Simple and complex movements in a patient with infarction of the right supplementary motor area. Mov Disord 1986; 1: 255-266.

6. Duffau, H., Capelle, L., Denvil, D., et al.: The role of dominant premotor cortex in language: a study using intraoperative functional mapping in awake patients. Neuroimage 2003; 20: 1903-1914.

7. Fontaine, D., Capelle, L., Duffau, H.: Somatotopy of the supplementary motor area: evidence from correlation of the extent of surgical resection with the clinical patterns of deficit. Neurosurgery 2002; 50: 297-303.

8. Freund, H.J.: Functional organization of the human supplementary motor area and dorsolateral premotor cortex. Adv Neurol 1996; 70: 263-269.

9. Freund, H.J., Hummelsheim, H.: Lesions of premotor cortex in man. Brain 1985; 108: 697-733.

10. Halsband, U., Ito, N., Tanji, J., Freund, H.J.: The role of premotor cortex and the supplementary motor area in the temporal control of movement in man. Brain 1993; 116: 243266.

11. Hanakawa, T., Honda, M., Sawamoto, N., et al.: The role of rostral Brodmann area 6 in mental-operation tasks: an integrative neuroimaging approach. Cereb Cortex 2002; 12: 1157-1170.

12. Helmstaedter, C., Gleissner, U., Zentner, J., Elger, 
C.E.: Neuropsychological consequences of epilepsy surgery in frontal lobe epilepsy. Neuropsychologia 1998; 36: 681-689.

13. Krainik, A., Duffau, H., Capelle, L., et al.: Role of the healthy hemisphere in recovery after resection of the supplementary motor area. Neurology 2004; 62: 1323-1332.

14. Krainik, A., Lehericy, S., Duffau, H. et al.: Postoperative speech disorder after medial frontal surgery: role of the supplementary motor area. Neurology 2003; 60: 587-594.

15. Krainik, A., Lehericy, S., Duffau, H., et al.: Role of the supplementary motor area in motor deficit following medial frontal lobe surgery. Neurology 2001; 57: 871-878.

16. Krings, T., Buchbinder, B.R., Butler, W.E., et al.: Functional magnetic resonance imaging and transcranial magnetic stimulation: complementary approaches in the evaluation of cortical motor function. Neurology 1997; 48: 1406-1416.

17. Laplane, D., Talairach, J., Meininger, V., Bancaud, J., Orgogozo, J.M.: Clinical consequences of corticectomies involving the supplementary motor area in man. J Neurol Sci 1977; 34: 301-314.

18. Lim, S.H., Dinner, D.S., Pillay, P.K. et al.: Functional anatomy of the human supplementary sensorimotor area: results of extraoperative electrical stimulation. Electroencephalogr Clin Neurophysiol 1994; 91: 179-193.

19. Luders, H.O.: The supplementary sensorimotor area. An overview. Adv Neurol. 1996; 70: 1-16.

20. Luppino, G., Matelli, M., Camarda, R., Rizzolatti, G.: Corticocortical connections of area F3 (SMA-proper) and area F6 (pre-SMA) in the macaque monkey. J Comp Neurol 1993; 338: 114-140.

21. Masdeu, J.C., Schoene, W.C., Funkenstein, H.: Aphasia following infarction of the left supplementary motor area: a clinicopathologic study. Neurology 1978 ; 28 :1220-1223.

22. Nelson, L., Lapsiwala, S., Haughton, V.M. et al.: Preoperative mapping of the supplementary motor area in patients harboring tumors in the medial frontal lobe. J Neurosurg 2002; 97: 1108-1114.

23. Pai, M.C.: Supplementary motor area aphasia: a case report. Clin Neurol Neurosurg 1999; 101: 29-32.

24. Penfield, W.: The supplementary motor area in the cerebral cortex of man. Arch Psychiatr Nervenkr Z Gesamte Neurol Psychiatr 1950; 185: 670-674.

25. Penfield, W.: Mechanisms of voluntary movement. Brain 1954; 77: 1-17.

26. Penfield, W., Welch, K.: The supplementary motor area of the cerebral cortex; a clinical and experimental study. AMA Arch Neurol Psychiatry 1951; 66: 289-317.

27. Peraud, A., Meschede, M., Eisner, W., Ilmberger, J., Reulen, H.J.: Surgical resection of grade II astrocytomas in the superior frontal gyrus. Neurosurgery 2002; 50: 966-975.

28. Rizzolatti, G., Fadiga, L., Matelli, M., et al.: Localization of grasp representations in humans by PET: 1. Observation versus execution. Exp Brain Res 1996; 111: 246-252.

29. Rogers, B.P., Carew, J.D., Meyerand, M.E.: Hemis- pheric asymmetry in supplementary motor area connectivity during unilateral finger movements. Neuroimage 2004; 22: 855-859.

30. Ross, E.D., Harney, J.H., Lacoste-Utamsing, C., Purdy, P.D.: How the brain integrates affective and propositional language into a unified behavioral function. Hypothesis based on clinicoanatomic evidence. Arch Neurol 1981; 38: 745-748.

31. Rostomily, R.C., Berger, M.S., Ojemann, G.A., Lettich, E.: Postoperative deficits and functional recovery following removal of tumors involving the dominant hemisphere supplementary motor area. J Neurosurg 1991; 75: 62-68.

32. Rubens, A.B.: Aphasia with infarction in the territory of the anterior cerebral artery. Cortex 1975; 11: 239-250.

33. Sailor, J., Meyerand, M.E., Moritz, C.H., et al.: Supplementary motor area activation in patients with frontal lobe tumors and arteriovenous malformations. AJNR Am J Neuroradiol 2003; 24: 1837-1842.

34. Solodkin, A., Hlustik, P., Noll, D.C., Small, S.L.: Lateralization of motor circuits and handedness during finger movements. Eur J Neurol 2001; 8: 425-434.

35. Tanji, J., Okano, K., Sato, K.C.: Neuronal activity in cortical motor areas related to ipsilateral, contralateral, and bilateral digit movements of the monkey. J Neurophysiol 1988; 60: 325-343.

36. Tanji, J., Shima, K.: Role for supplementary motor area cells in planning several movements ahead. Nature 1994; 371 : 413-416.

37. Vitaz, T.W., Marx, W., Victor, J.D., Gutin, P.H.: Comparison of conscious sedation and general anesthesia for motor mapping and resection of tumors located near motor cortex. Neurosurg Focus 2003; 15: 8.

38. Yamane, F., Muragaki, Y., Maruyama, T. et al.: Preoperative mapping for patients with supplementary motor area epilepsy: multimodality brain mapping. Psychiatry Clin Neurosci 2004; 58: 16-21.

39. Zentner, J., Hufnagel, A., Pechstein, U., Wolf, H.K., Schramm, J.: Functional results after resective procedures involving the supplementary motor area. J Neurosurg 1996; 85: 542-549.

40. Ziegler, W., Kilian, B., Deger, K.: The role of the left mesial frontal cortex in fluent speech: evidence from a case of left supplementary motor area hemorrhage. Neuropsychologia 1997; 35: 1197-1208.

Gabarrós, A.; Martino, J.; Juncadella, M.; Plans, G.; Pujol, R; Deus, J.; Godino, O.; Torres, A.; Aparicio, A.; Conesa, G.; Acebes, J.J.: Identificación intraoperatoria del área motora suplementaria en cirugía neurooncológica. Neurocirugía 2011; 22: 123-132.

Correspondencia: Hospital Universitari de Bellvitge. Feixa Llarga s/n. 08907 L'Hospitalet de Llobregat (Barcelona). e-mail: agabarros@ub.edu 